\title{
Changes in sagittal alignment after surgical excision of thoracic spinal cord tumors in adults
}

\author{
Yoshiomi Kobayashi ${ }^{1,2} \cdot$ Soya Kawabata ${ }^{2,3} \cdot$ Yuichiro Nishiyama $^{2,4} \cdot$ Osahiko Tsuji $^{1,2} \cdot$ Eijiro Okada ${ }^{1,2}$. \\ Nobuyuki Fujita $^{1,2} \cdot$ Mitsuru Yagi $^{1,2} \cdot$ Kota Watanabe $^{1,2} \cdot$ Morio Matsumoto $^{1,2} \cdot$ Masaya Nakamura $^{1,2}$. \\ Narihito Nagoshi ${ }^{1,2}$
}

Received: 10 September 2018 / Revised: 4 December 2018 / Accepted: 10 December 2018 / Published online: 8 January 2019

(C) International Spinal Cord Society 2019

\begin{abstract}
Study design Retrospective chart audit.

Objectives This study investigated changes in sagittal alignment in adults after excision of thoracic spinal cord tumors without spinal fixation.

Setting Single-center study at an academic orthopedic department in Japan.

Methods We retrospectively reviewed records for 32 adults who underwent excision of thoracic spinal cord tumors by multilevel laminectomies without fixation. The participants were divided according to whether the tumor was in the upper (T1-4), middle (T5-8), or lower (T9-12) thoracic spine. We analyzed parameters such as age, sex, time in surgery and estimated blood loss, follow-up period, and preoperative and follow-up the Japanese Orthopaedic Association (JOA) scores and radiographs.

Results Postoperative T1-12 kyphotic changes did not correlate with age, the number of resected laminae, or preoperative T1-12 kyphosis. JOA recovery rates were similar regardless of the tumor location. Participants with tumors in the upper thoracic spine had significant postoperative increases in T1-4 kyphosis, T1 slope ( $p<.05$, respectively). In contrast, there were no significant changes in alignment in participants with tumors in the middle or lower thoracic spine.

Conclusion Even without fixation, sagittal alignment did not change after surgery to excise tumors in the middle and lower thoracic spine, indicating that fixation may not be necessary when excising spinal cord tumors in this region. In contrast, postoperative kyphosis may increase when the tumor is in the upper thoracic spine.
\end{abstract}

\section{Introduction}

Laminectomy is a common procedure for decompressing the spinal cord and directly removing tumors or other

Supplementary information The online version of this article (https:// doi.org/10.1038/s41393-018-0235-3) contains supplementary material, which is available to authorized users.

Narihito Nagoshi

nagoshi@2002.jukuin.keio.ac.jp

1 Department of Orthopaedic Surgery, Keio University School of Medicine, Tokyo, Japan

2 Keio Spine Research Group (KSRG), Tokyo, Japan

3 Department of Orthopaedic Surgery, Keiyu Hospital, Kanagawa, Yokohama, Japan

4 Department of Orthopaedic Surgery, Saitama City Hospital, Saitama, Japan matter that is causing compression. Although laminectomies are used to treat several cervical and thoracic spinal diseases $[1,2]$, they can cause spinal instability leading to progressive kyphosis [2-5]. Kyphosis in the spinal alignment can lead to neurological deterioration, pain, and problems with posture [4, 5]. Postoperative kyphosis has mostly been documented in children and may be associated with the number and level of the resected laminae [5-7]. In the thoracic spine, progressive malalignment is observed mostly in multilevel laminectomies in children [5-8]. Although studies have concluded that the stability of the thoracic cage in adults is sufficient to prevent severe instability or deformity after laminectomy in the thoracic spine [9-11], these studies examined cases of spondylosis and ossification of the posterior longitudinal ligament (OPLL), not spinal cord tumors, and evaluated spinal alignment only at the decompressed levels. The impact of thoracic laminectomy 
on global spinal alignment in adults with spinal cord tumors has not been determined.

Spinal cord tumors represent $0.5 \%$ of newly diagnosed neoplasms and $5-12 \%$ of all tumors of the central nervous system [12]. Spinal cord tumors are less common in adults than in children [13]. Excising a spinal cord tumor often requires several wide laminectomies to open up enough space to resect the tumor safely, and the laminectomies can lead to instability and subsequent kyphosis due to damage to the posterior elements of the spinal column [5, 6, 14]. Although spinal instrumentation and fusion can prevent spinal deformity after the surgical excision of intramedullary spinal cord tumors $[15,16]$, instrumentation has disadvantages. First, metallic artifacts from the instrumentation make it difficult to confirm the status of the surgical site after surgery or to check for tumor recurrence by magnetic resonance imaging (MRI). Second, instrumentation can interfere with postoperative radiotherapy [17]. Third, instrumentation increases the possibility of meningitis if a cerebrospinal fluid leak occurs with wound infection [18]. Finally, instrumentation increases the total cost of surgery and the expense of hospitalization [19]. Thus, decompression without fixation is preferable when resecting thoracic spinal cord tumors. However, to date there are no reports on the long-term effect of thoracic spinal cord tumor resection on the sagittal alignment of the entire spine in adults.

In the present study, we retrospectively reviewed clinical outcomes for adults who underwent surgical excision of spinal cord tumors at the thoracic level, and investigated which factors could be associated with postoperative sagittal alignment change. This is the first study to examine how excision surgery using laminectomy without fixation affects sagittal alignment in adults with these rare neoplastic diseases.

\section{Methods}

\section{Persons and clinical assessments}

We retrospectively reviewed records for 76 individuals who underwent surgical excision of thoracic spinal cord tumor with multilevel thoracic laminectomies between January 2012 and April 2015 at a teaching hospital. Individuals were eligible for inclusion in the study if they were at least 20 years old, had laminectomies at two or more levels, did not have a facetectomy, were followed at least 12 months, and had whole-spine standing radiographs obtained prior to surgery and at the final follow-up. Individuals were excluded if they underwent postoperative radiotherapy or had a dumbbell-shaped tumor. The 32 individuals who met the criteria for the study were grouped by whether the tumor was located in the upper thoracic (T1-4), middle thoracic
(T5-8), or lower thoracic spine (T9-12), as previously reported for assessing OPLL and thoracic spondylosis [9]. The following data were recorded for each individual: age, sex, estimated blood loss (EBL), time in surgery, follow-up period, and radiological findings. Neurological status was evaluated prior to surgery and at the final follow-up with the Japanese Orthopaedic Association (JOA) scoring system (11 possible points). The recovery rate was calculated as (postoperative JOA score-preoperative JOA score)/(11 - preoperative JOA score $) \times 100(\%)$.

\section{Radiological parameters}

Radiological parameters were measured from lateral whole-spine standing radiographs, obtained prior to surgery and at the final follow-up, as described previously [20]. Briefly, we measured the following: the angle subtended by a perpendicular from the upper endplate of $\mathrm{S} 1$ and a line connecting the center of the femoral head to the center of the upper endplate of S1 (pelvic incidence, PI); the angle between the inferior endplate of T12 and the superior endplate of S1 using the Cobb method (lumbar lordosis, LL); the angle formed by a line drawn along the endplate of the sacrum and a horizontal reference line (sacral slope, $\mathrm{SS}$ ); the distance between the $\mathrm{C} 7$ plumb line and the posterior corner of the sacrum (C7-sagittal vertical axis, C7 SVA); thoracic kyphosis (TK, T1-T12), measured as the angle between the inferior endplate of $\mathrm{C} 7$ and the superior endplate of T12 using the Cobb method; the angle between the inferior endplate of $\mathrm{C} 7, \mathrm{~T} 4$, and $\mathrm{T} 8$ and the superior endplate of $\mathrm{T} 4, \mathrm{~T} 8$, and $\mathrm{T} 12$, respectively, using the Cobb method (T1-4, T5-8, and T9-12); the $\mathrm{T} 1$ slope, measured as the angle formed by a line drawn along the superior endplate of the $\mathrm{T} 1$ and a horizontal reference line; and the $\mathrm{C} 2-7$ angle, measured between the posterior surface of $\mathrm{C} 2$ and the posterior surface of C7. The PI was used as an invariable morphologic parameter for each individual, and other parameters were measured before and after the operation without reference to the PI [21].

\section{Statistical methods}

Values were expressed as the mean \pm standard deviation. Differences between pre- and postoperative radiological parameters were assessed using Student's $t$-test when the individuals were grouped according to the surgical technique. Non-normally distributed variables were compared by the Kurskal-Wallis test. If the Kurskal-Wallis test showed statistical significance, Dunn's multiple comparison test was used for further analysis. Correlations between parameters were calculated using Spearman's rank correlation coefficients. A $p$ value less than .05 was considered statistically significant. We 
used G*Power software (version 3.1.9.3; Heinrich Heine University Dusseldorf, Germany) for the power analysis. GraphPad Prism software (version 6.0c; GraphPad Software Inc., CA, USA) was used for the other analyses.

Table 1 Demographics

\begin{tabular}{ll}
\hline Number of cases & 32 \\
Age (years) & $54.4 \pm 17.8$ \\
Sex (M: F) & $18: 14$ \\
Follow-up (months) & $24.8 \pm 11.4$ \\
EBL (mL) & $136 \pm 145$ \\
Operating time (min) & $287 \pm 107$ \\
Number of resected laminae & $3.59 \pm 0.88$ \\
Type of tumor & \\
Intradural extramedullary tumor & 19 (12 neurinoma, 7 hemangioma) \\
Intramedullary tumor & 13 (5 ependymoma, 5 hemangioma, 2 lipoma, \\
& 1 ependymal cyst) \\
Surgical procedure & LAM 22, SPSL 10 \\
& Mean \pm standard deviation
\end{tabular}

EBL estimated blood loss, LAM laminectomy, SPSL spinous processsplitting laminectomy

\section{Results}

This study included 18 men and 14 women with an average age of $54.4 \pm 17.8$ years (range $21-83$ years). The mean clinical and radiological follow-up was $24.8 \pm 11.4$ months (range 11-52 months). The mean EBL was $136 \pm 145 \mathrm{~mL}$ (range $50-450 \mathrm{~mL}$ ), the mean time in surgery was $287 \pm$ $107 \mathrm{~min}$ (range 118-605 $\mathrm{min}$ ), and the mean number of resected laminae was $3.59 \pm 0.88$ (range 2-6 laminae). Regarding the tumor type, 19 individuals were treated for intradural extramedullary tumors and 13 for intramedullary tumors. The operation included spinous process-splitting laminectomy (SPSL) in 10 cases and laminectomy without splitting (LAM) in 22 cases (Table 1). Supplementary Table 1 summarizes the clinical data for all individuals.

We analyzed several factors that could be associated with a postoperative sagittal alignment change. There was no correlation between a postoperative increase in T1-12 kyphosis and the person's age $(r=0.0046, p=.97)$, the number of resected laminae $(r=-0.11, p=.56)$, or the preoperative angle of T1-12 kyphosis $(r=0.054, p=.77)$
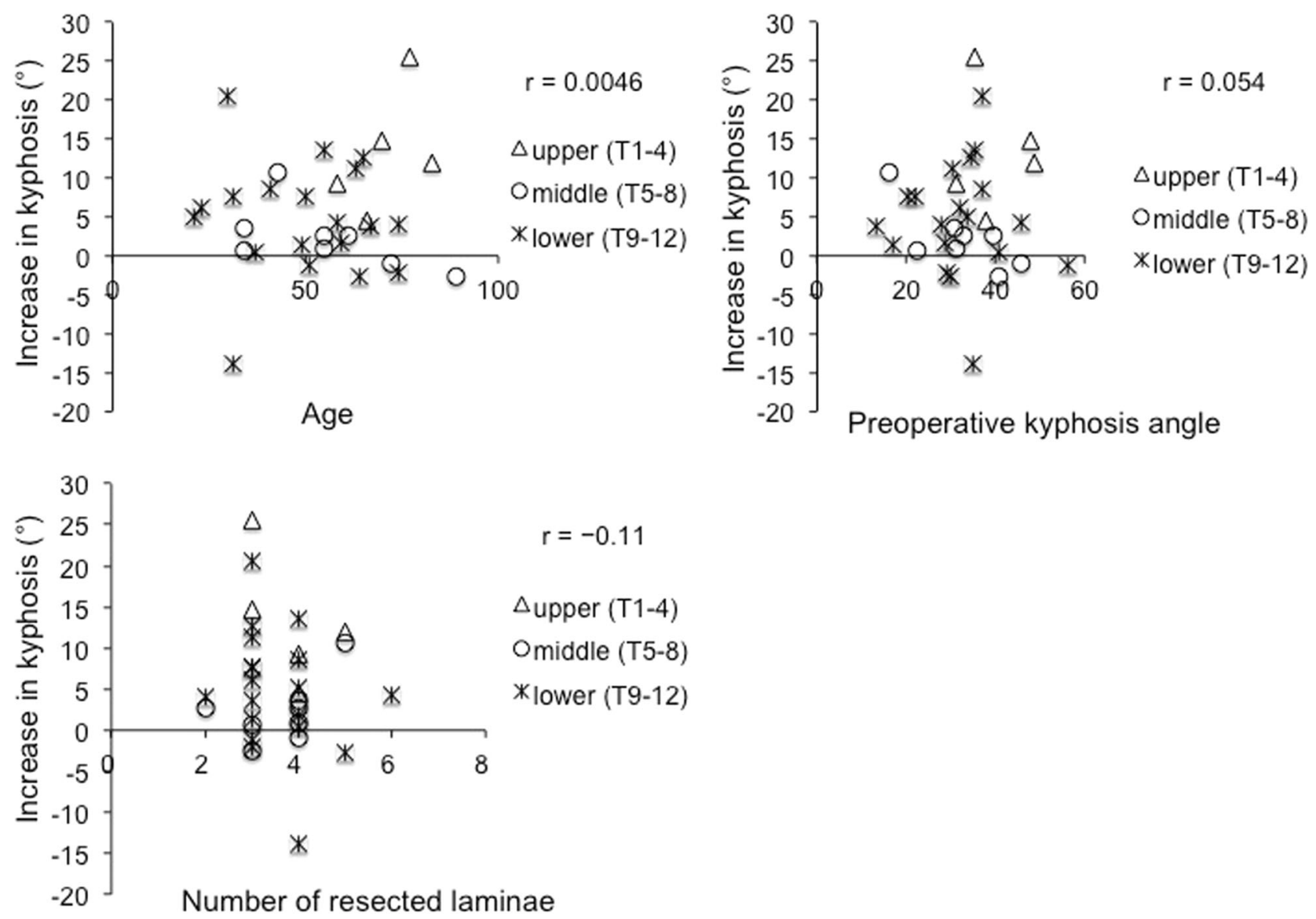

Fig. 1 Correlation between postoperative kyphotic changes with age, the number of resected laminae, or preoperative kyphosis. There was no correlation between age and a postoperative increase in the T1-12 kyphotic angle $(r=0.0046)$ and between the number of resected

laminae and a postoperative increase in the T1-12 kyphotic angle $(r=-0.11)$. The angle of the T1-12 kyphosis before surgery did not correlate with a postoperative increase in the T1-12 kyphotic angle $(r=0.054)$ 
when we included all the persons in the analysis, regardless of the tumor location (see Fig. 1). When the individuals were grouped by tumor location, there were five upper thoracic, eight middle thoracic, and 19 lower thoracic tumors, and there was no significant difference between these groups in age (upper thoracic $70.8 \pm 9.7$ years, middle thoracic 55.4 \pm 18.9 , and lower thoracic 49.6 \pm 16.9 ; $p=.054)$ or type of tumor $(p=.46)$. The JOA recovery rates were similar in the three groups (upper thoracic $13.3 \%$, middle thoracic $31.8 \%$, and lower thoracic $23.9 \%$; $p=.44$ ); see Fig. 2. Table 2 shows the sagittal alignment changes according to tumor distribution. There were significant increases in T1-4 kyphosis and T1 slope in the upper thoracic group compared to the other groups $(p<.05$, respectively). Moreover, T1-12 kyphosis was significantly increased in the upper thoracic group compared to the middle thoracic group $(p<.05)$. T5-8 kyphosis in the upper

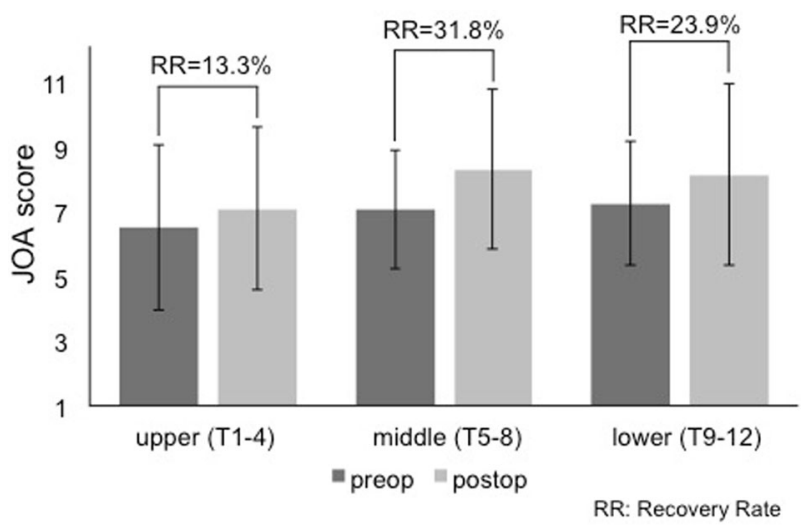

Fig. 2 Average rate of neurological recovery by tumor location. The JOA recovery rate according to tumor location in the thoracic spine: upper $13.3 \%$, middle $31.8 \%$, lower $23.9 \%$. These differences were not significant $(p=.44)$ thoracic group was significantly decreased compare to lower thoracic group $(p<.05)$ (Fig. 3). C2-7 lordosis in the upper thoracic group was more increased, although there was no statistically significant difference. A post-hoc power analysis was performed to assess the difference in T1-4 kyphosis between the three groups. The statistical power was 1.00. Next, we analyzed only the cases treated by LAM, of which there were five in the upper thoracic, four in the middle thoracic, and 13 in the lower thoracic spine. There were significant increases in the T1-4 kyphosis and T1 slope in the upper thoracic group compared to the lower thoracic group ( $p<.05$ for both). The T5-8 kyphosis in the upper thoracic group was significantly decreased compared to the lower thoracic group $(p<.05)$ (Supplementary Fig. 1). When individuals were grouped by the LAM or SPSL surgical technique, we did not find any changes between preoperative and postoperative sagittal alignment parameters except for the T1 slope, which increased significantly in the LAM group (from $19.9 \pm 7.36^{\circ}$ to $26.5 \pm$ $\left.11.3^{\circ}, p=.04\right)$; see Table 3 .

\section{Discussion}

The current study is the first to compare sagittal alignment in adults before and after the surgical excision of thoracic spinal cord tumors without fixation. Postoperative kyphosis has mostly been reported in children and may be associated with the number and level of the resected laminae [5-7]. Kimura et al. reported that persons with preoperative kyphotic alignment had worsened kyphosis after laminectomy in the cervical spine [22]. Although the sample size was not large in this study, we did not find any correlations between a postoperative increase in T1-12 kyphosis and the person's age, the number of resected
Table 2 Sagittal alignment changes according to tumor distribution

\begin{tabular}{|c|c|c|c|c|c|c|}
\hline & \multicolumn{2}{|l|}{ Upper } & \multicolumn{2}{|l|}{ Middle } & \multicolumn{2}{|l|}{ Lower } \\
\hline & Preop & Postop & Preop & Postop & Preop & Postop \\
\hline $\operatorname{PI}\left({ }^{\circ}\right)$ & $49.0 \pm 16.6$ & - & $52.6 \pm 11.0$ & - & $51.7 \pm 12.9$ & - \\
\hline $\operatorname{LL}\left({ }^{\circ}\right)$ & $43.2 \pm 13.5$ & $42.3 \pm 15.1$ & $38.1 \pm 11.0$ & $36.6 \pm 13.5$ & $42.1 \pm 11.1$ & $45.5 \pm 10.1$ \\
\hline $\mathrm{SS}\left({ }^{\circ}\right)$ & $28.3 \pm 10.7$ & $29.9 \pm 14.3$ & $27.2 \pm 9.48$ & $28.4 \pm 10.9$ & $28.4 \pm 7.99$ & $31.5 \pm 7.57$ \\
\hline C7 SVA (mm) & $13.1 \pm 18.4$ & $28.5 \pm 21.8$ & $-0.75 \pm 22.3$ & $13.0 \pm 17.8$ & $5.95 \pm 27.4$ & $6.37 \pm 26.0$ \\
\hline $\mathrm{T} 1-12\left(^{\circ}\right)$ & $40.1 \pm 7.74$ & $53.4 \pm 11.0$ & $32.5 \pm 9.77$ & $34.6 \pm 7.29$ & $31.8 \pm 9.97$ & $36.5 \pm 12.2$ \\
\hline $\mathrm{T} 1-4\left(^{\circ}\right)$ & $11.8 \pm 9.93$ & $31.9 \pm 12.9$ & $12.9 \pm 3.63$ & $13.9 \pm 5.45$ & $7.42 \pm 6.09$ & $8.47 \pm 5.86$ \\
\hline $\mathrm{T} 5-8\left(^{\circ}\right)$ & $24.7 \pm 9.85$ & $16.9 \pm 6.82$ & $14.9 \pm 6.47$ & $14.9 \pm 7.44$ & $12.9 \pm 6.71$ & $16.5 \pm 8.40$ \\
\hline T9-12 $\left(^{\circ}\right)$ & $3.66 \pm 10.7$ & $4.58 \pm 9.02$ & $4.68 \pm 4.56$ & $5.79 \pm 6.25$ & $11.4 \pm 9.47$ & $11.6 \pm 8.98$ \\
\hline T1 slope $\left({ }^{\circ}\right)$ & $25.1 \pm 5.72$ & $41.1 \pm 10.4$ & $22.1 \pm 7.45$ & $23.7 \pm 6.18$ & $17.8 \pm 8.44$ & $20.2 \pm 7.53$ \\
\hline $\mathrm{C} 2-7\left(^{\circ}\right)$ & $18.5 \pm 6.35$ & $32.9 \pm 9.93$ & $10.5 \pm 8.67$ & $10.7 \pm 9.79$ & $10.7 \pm 13.9$ & $11.7 \pm 13.6$ \\
\hline
\end{tabular}

Mean \pm standard deviation

$P I$ pelvic incidence, $L L$ lumbar lordosis, $S S$ sacral slope, $S V A$ sagittal vertical axis, $T K$ thoracic kyphosis 

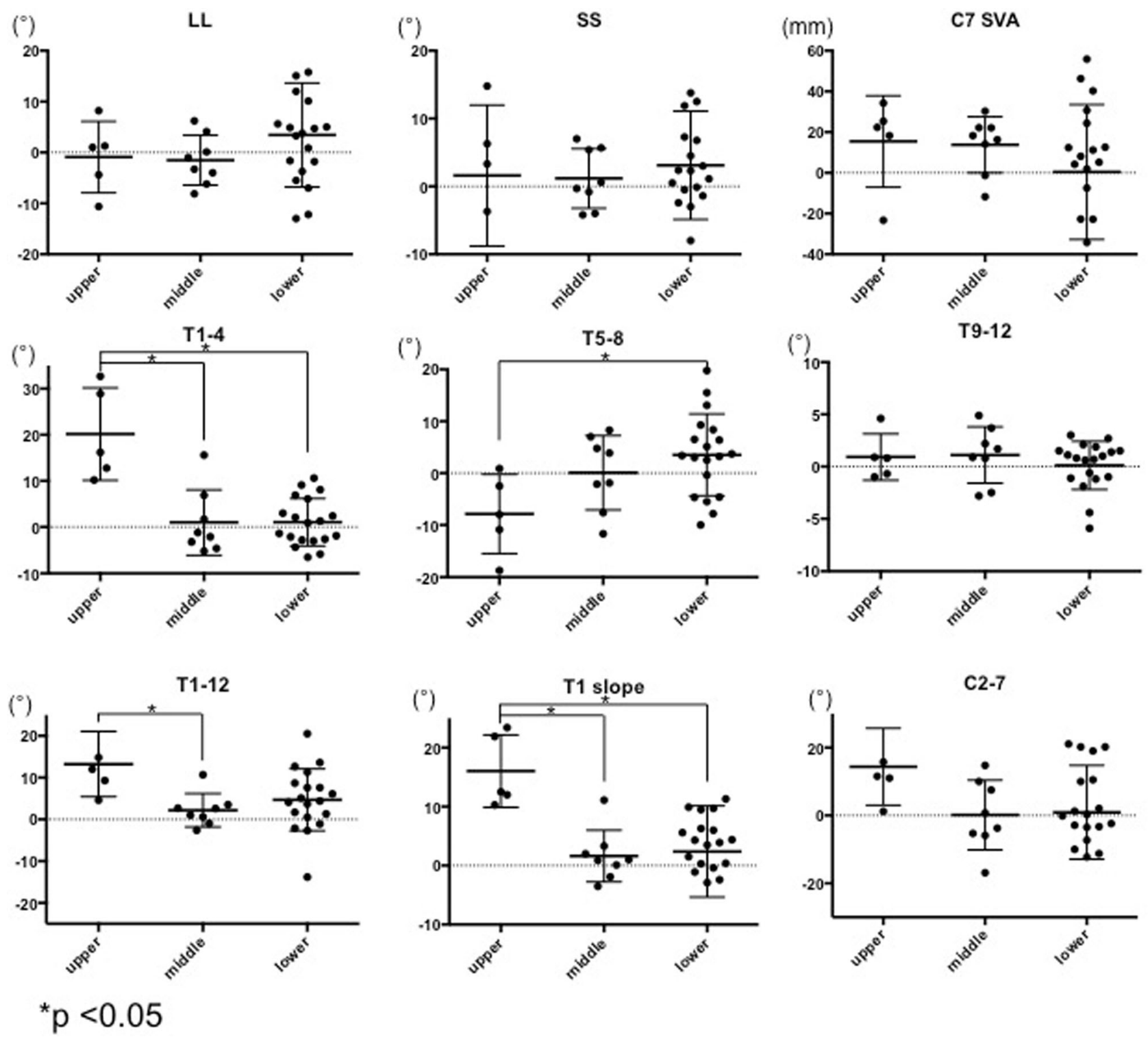

Fig. 3 Postoperative sagittal alignment changes according to tumor distribution. There were significant increases in T1-4 kyphosis and $\mathrm{T} 1$ slope in the upper thoracic group compare to the other groups. Moreover, T1-12 kyphosis was significantly increased in the upper

thoracic group compared to the middle thoracic group. T5-8 kyphosis in upper group was significantly decreased compare to lower thoracic group

laminae, or the preoperative T1-12 kyphosis, when we performed the analysis for all persons regardless of tumor location. Even if persons were grouped by tumor location, there were no significant changes between preoperative and postoperative sagittal alignment parameters in persons with spinal cord tumors in the middle thoracic or lower thoracic spine. One study reported that in persons with thoracic spinal cord tumors, those with more than three laminae removed developed spinal deformities more rapidly than did those with fewer resected laminae [23]. In particular, involvement of the thoracolumbar junction increases the risk that spinal alignment will deteriorate after surgery
[7, 8, 23]. However, these studies examined children, whereas our study assessed adults. Even in adults with spinal cord tumors in the lower thoracic region, which includes the thoracolumbar junction, we did not observe any significant change in the sagittal alignment after excision surgery. Therefore, our results suggest that spinal fixation may not be mandatory for adults with spinal cord tumors at the middle or lower thoracic levels.

In contrast, kyphosis increased after surgery in persons with spinal cord tumors in the upper thoracic spine, who showed postoperative increases in both the T1-4 kyphosis and $\mathrm{T} 1$ slope. The upper thoracic levels (T1-4) are included 
Table 3 Sagittal alignment changes according to surgical procedure

\begin{tabular}{|c|c|c|c|c|c|c|}
\hline & \multicolumn{3}{|l|}{ LAM } & \multicolumn{3}{|l|}{ SPSL } \\
\hline & Preop & Postop & $p$ & Preop & Postop & $p$ \\
\hline $\mathrm{PI}\left({ }^{\circ}\right)$ & $53.8 \pm 13.0$ & - & - & $46.3 \pm 11.0$ & - & - \\
\hline $\operatorname{LL}\left({ }^{\circ}\right)$ & $41.8 \pm 10.6$ & $42.3 \pm 12.5$ & 0.83 & $40.1 \pm 13.0$ & $43.8 \pm 11.5$ & 0.64 \\
\hline $\mathrm{SS}\left({ }^{\circ}\right)$ & $28.1 \pm 8.70$ & $31.1 \pm 9.74$ & 0.12 & $28.1 \pm 8.50$ & $29.1 \pm 8.86$ & 0.93 \\
\hline C7 SVA (mm) & $2.66 \pm 20.6$ & $16.7 \pm 22.6$ & 0.08 & $11.4 \pm 32.4$ & $0 \pm 24.9$ & 0.36 \\
\hline $\mathrm{T} 1-12\left(^{\circ}\right)$ & $34.2 \pm 9.87$ & $40.3 \pm 12.6$ & 0.12 & $31.3 \pm 9.88$ & $35.1 \pm 12.1$ & 0.47 \\
\hline $\mathrm{T} 1-4\left(^{\circ}\right)$ & $10.4 \pm 7.09$ & $15.8 \pm 12.0$ & 0.17 & $7.37 \pm 5.14$ & $8.35 \pm 4.93$ & 0.52 \\
\hline $\mathrm{T} 5-8\left(^{\circ}\right)$ & $16.0 \pm 9.13$ & $16.6 \pm 8.51$ & 0.70 & $13.6 \pm 5.29$ & $15.1 \pm 5.99$ & 0.72 \\
\hline T9-12 $\left(^{\circ}\right)$ & $7.72 \pm 10.4$ & $7.82 \pm 9.40$ & 0.99 & $10.3 \pm 5.53$ & $11.7 \pm 6.61$ & 0.53 \\
\hline T1 slope $\left(^{\circ}\right)$ & $19.9 \pm 7.36$ & $26.5 \pm 11.3$ & $0.04 *$ & $20.4 \pm 10.1$ & $19.6 \pm 7.07$ & 0.84 \\
\hline $\mathrm{C} 2-7\left(^{\circ}\right)$ & $14.8 \pm 9.64$ & $18.4 \pm 13.9$ & 0.41 & $5.43 \pm 14.4$ & $6.6 \pm 12.3$ & 0.62 \\
\hline
\end{tabular}

Mean \pm standard deviation

LAM laminectomy, SPSL spinous process-splitting laminectomy

$P I$ pelvic incidence, $L L$ lumbar lordosis, $S S$ sacral slope, $S V A$ sagittal vertical axis, $T K$ thoracic kyphosis in the cervical-thoracic vertebral transition (CTJ), which is in the region from $\mathrm{C} 7$ to $\mathrm{T} 3 / 4$, and the CTJ is strongly correlated with postoperative instability due to its anatomical features [24, 25]. In this region, transition from the mobile, articulated cervical spine, which is lordotic, to the rigid, kyphotic thoracic spine creates a mechanical vulnerability that may be amplified after surgery [26]. It is likely that these anatomical characteristics could contribute to the progressive kyphosis that we observed in persons with laminectomies in the upper thoracic spine, and that a subsequent compensatory lordotic change in the cervical spine and decrease in kyphosis in the middle thoracic spine might occur to avoid sagittal imbalance. To prevent this postoperative change in alignment, Avila et al. proposed criteria for stabilization and fusion after spinal cord tumor resection in adults [27]. Avila et al. also recommended that persons who develop postoperative deformities undergo conservative treatment for at least six months, followed by spinal fixation if the symptoms persist or worsen. In the present study, people were followed about two years on average and did not report severe symptoms or neurological deterioration despite kyphotic changes. However, individuals with postoperative changes in sagittal alignment should be observed carefully, especially with regard to thoracic alignment, and fixation should be considered as a second-stage surgery if the kyphosis progresses without appropriate compensation in the cervical and middle thoracic spine.

SPSL helps to preserve spinal alignment by preserving posterior elements such as muscles, facet capsules, and interspinous and supraspinous ligaments [28, 29]. In the present study, none of the people treated by SPSL showed any change between preoperative parameters and those measured at the final follow-up (Table 3). On the other hand, the T1 slope increased significantly after surgery in persons treated by LAM. It should be noted that all persons with tumors in the upper thoracic spinal cord were treated by LAM, and it is possible that this particular technique influenced the progression of T14 kyphosis and $\mathrm{T} 1$ slope over time. If this were the case, SPSL might be preferable when removing spinal cord tumors at the upper thoracic levels. However, the T1-4 kyphosis and T1 slope significantly increased in the upper thoracic group compared to the lower thoracic group when we analyzed only the persons treated by LAM. This result suggested that differences in the surgical procedure between the three groups were not responsible for the results of this study. To clarify the possible effect of the surgical method on postoperative kyphosis, more persons treated SPSL for tumors in the in the upper thoracic region will need to be assessed. In addition, osteoporotic compression deformities or subsequent kyphotic spinal changes might progress with increasing age, because the vertebrae were left with impaired posterior tension bands by the laminectomy, even in the lower thoracic or thoracolumbar region. These changes would be detected with longer follow-up periods. Although no persons treated by hemilaminectomy were included in this study, previous studies reported that the average change in the kyphosis angle was greater in a LAM group than in a hemilaminectomy group [30]. Thus, further study is still needed to confirm the best methods for avoiding postoperative kyphosis.

This study has several limitations. First, the retrospective study design has a lower evidence level. Second, the small sample size reduces statistical power and limits us to broad generalizations from the current results. Third, we evaluated posture only through radiographic measurements; a prospective study would allow a more comprehensive evaluation of postural deformities before and after surgery. Lastly, this study lacks a control group treated by laminectomy for 
a different thoracic spinal disorder (i.e., spondylosis). The analysis of a matched case-control study is still needed to investigate the influence of the spinal cord tumor itself. However, spinal cord tumors are relatively rare compared to other spinal diseases, and our findings may provide a useful reference when considering surgical techniques and their influence on postoperative global spinal alignment.

\section{Data archiving}

The datasets generated and/or analyzed during the current study are available from the corresponding author on request.

Acknowledgements We are grateful to the individuals who participated in this study. We also thank Dr. Takahiro Tadokoro for advice on statistical analyses.

Author contributions: YK collected, and interpreted the data and wrote the initial draft of the manuscript. SK and YN collected data. OT, EO, NF, MY, KW assisted in the preparation of the manuscript. $\mathrm{MM}, \mathrm{MN}, \mathrm{NN}$ designed the study, and critically reviewed the manuscript. All authors approved the final version of the manuscript, and agreed to be accountable for all aspects of the work in ensuring that questions related to the accuracy or integrity of any part of the work were appropriately investigated and resolved.

\section{Compliance with ethical standards}

Conflict of interest The authors declare that they have no conflict of interest.

Ethical approval We certify that all applicable institutional and governmental regulations concerning the ethical use of human volunteers were followed during the course of this research.

Publisher's note: Springer Nature remains neutral with regard to jurisdictional claims in published maps and institutional affiliations.

\section{References}

1. Aizawa T, Sato T, Sasaki H, Matsumoto F, Morozumi N, Kusakabe $\mathrm{T}$, et al. Results of surgical treatment for thoracic myelopathy: minimum 2-year follow-up study in 132 patients. J Neurosurg Spine. 2007;7:13-20.

2. Herkowitz HN. A comparison of anterior cervical fusion, cervical laminectomy, and cervical laminoplasty for the surgical management of multiple level spondylotic radiculopathy. Spine. 1988;13:774-80.

3. Fong SY, Wong HK. Thoracic myelopathy secondary to ligamentum flavum ossification. Ann Acad Med Singap. 2004; 33:340-6.

4. Mikawa Y, Shikata J, Yamamuro T. Spinal deformity and instability after multilevel cervical laminectomy. Spine. 1987; 12:6-11.

5. Yasuoka S, Peterson HA, MacCarty CS. Incidence of spinal column deformity after multilevel laminectomy in children and adults. J Neurosurg. 1982;57:441-5.

6. Papagelopoulos PJ, Peterson HA, Ebersold MJ, Emmanuel PR, Choudhury SN, Quast LM. Spinal column deformity and instability after lumbar or thoracolumbar laminectomy for intraspinal tumors in children and young adults. Spine. 1997;22:442-51.

7. Yao KC, McGirt MJ, Chaichana KL, Constantini S, Jallo GI. Risk factors for progressive spinal deformity following resection of intramedullary spinal cord tumors in children: an analysis of 161 consecutive cases. J Neurosurg. 2007;107:463-8.

8. Joaquim AF, Cheng I, Patel AA. Postoperative spinal deformity after treatment of intracanal spine lesions. Spine J. 2012; 12:1067-74.

9. Aizawa T, Sato T, Ozawa H, Morozumi N, Matsumoto F, Sasaki $\mathrm{H}$, et al. Sagittal alignment changes after thoracic laminectomy in adults. J Neurosurg Spine. 2008;8:510-6.

10. Lonstein JE. Post-laminectomy kyphosis. Clin Orthop Relat Res. 1977;128:93-100.

11. Miyazaki K, Kirita Y. Extensive simultaneous multisegment laminectomy for myelopathy due to the ossification of the posterior longitudinal ligament in the cervical region. Spine. 1986;11:531-42.

12. Fehlings MG, Chua SY. Editorial: spinal cord tumor research. J Neurosurg Spine. 2010;12:115-6.

13. Bowers DC, Weprin BE. Intramedullary spinal cord tumors. Curr Treat Options Neurol. 2003;5:207-12.

14. Wiggins GC, Shaffrey CI. Dorsal surgery for myelopathy and myeloradiculopathy. Neurosurgery. 2007;60:S71-81.

15. Simon SL, Auerbach JD, Garg S, Sutton LN, Telfeian AE, Dormans JP. Efficacy of spinal instrumentation and fusion in the prevention of postlaminectomy spinal deformity in children with intramedullary spinal cord tumors. J Pediatr Orthop. 2008; 28:244-9.

16. Anakwenze OA, Auerbach JD, Buck DW, Garg S, Simon SL, Sutton LN, et al. The role of concurrent fusion to prevent spinal deformity after intramedullary spinal cord tumor excision in children. J Pediatr Orthop. 2011;31:475-9.

17. Landmann C, Hunig R, Gratzl O. The role of laminectomy in the combined treatment of metastatic spinal cord compression. Int $\mathrm{J}$ Radiat Oncol Biol Phys. 1992;24:627-31.

18. Cummings RJ. Recurrent meningitis secondary to infection after spinal arthrodesis with instrumentation. A case report. J Bone Jt Surg Am. 1998;80:722-4.

19. Warren DT, Ricart-Hoffiz PA, Andres TM, Hoelscher CM, Protopsaltis TS, Goldstein JA, et al. Retrospective cost analysis of cervical laminectomy and fusion versus cervical laminoplasty in the treatment of cervical spondylotic myelopathy. Int J Spine Surg. 2013;7:e72-80.

20. Jackson RP, McManus AC. Radiographic analysis of sagittal plane alignment and balance in standing volunteers and patients with low back pain matched for age, sex, and size. A prospective controlled clinical study. Spine. 1994;19:1611-8.

21. Duval-Beaupere G, Schmidt C, Cosson P. A Barycentremetric study of the sagittal shape of spine and pelvis: the conditions required for an economic standing position. Ann Biomed Eng. 1992;20:451-62.

22. Kimura I, Shingu H, Nasu Y. Long-term follow-up of cervical spondylotic myelopathy treated by canal-expansive laminoplasty. J Bone Jt Surg Br. 1995;77:956-61.

23. Yeh JS, Sgouros S, Walsh AR, Hockley AD. Spinal sagittal malalignment following surgery for primary intramedullary tumours in children. Pediatr Neurosurg. 2001;35:318-24.

24. Wang VY, Chou D. The cervicothoracic junction. Neurosurg Clin N Am. 2007;18:365-71.

25. Steinmetz MP, Miller J, Warbel A, Krishnaney AA, Bingaman W, Benzel EC. Regional instability following cervicothoracic junction surgery. J Neurosurg Spine. 2006;4:278-84.

26. An HS, Vaccaro A, Cotler JM, Lin S. Spinal disorders at the cervicothoracic junction. Spine. 1994;19:2557-64. 
27. Avila MJ, Walter CM, Skoch J, Abbasifard S, Patel AS, Sattarov $\mathrm{K}$, et al. Fusion after intradural spine tumor resection in adults: A review of evidence and practices. Clin Neurol Neurosurg. 2015;138:169-73.

28. Watanabe K, Matsumoto M, Ikegami T, Nishiwaki Y, Tsuji T, Ishii $\mathrm{K}$, et al. Reduced postoperative wound pain after lumbar spinous process-splitting laminectomy for lumbar canal stenosis: a randomized controlled study. J Neurosurg Spine. 2011;14:51-8.
29. Shiraishi T, Kato M, Yato Y, Ueda S, Aoyama R, Yamane J, et al. New techniques for exposure of posterior cervical spine through intermuscular planes and their surgical application. Spine. 2012;37:E286-96.

30. Millward CP, Bhagawati D, Chan HW, Bestwick J, Brecknell JE. Retrospective observational comparative study of Hemilaminectomy versus Laminectomy for intraspinal tumour resection; shorter stays, lower analgesic usage and less kyphotic deformity. Br J Neurosurg. 2015;29:390-5. 